\title{
Monte Carlo Simulations of Polyion-Macroion Complexes. 1. Equal Absolute Polyion and Macroion Charges
}

\author{
Anna Akinchina* and Per Linse \\ Physical Chemistry 1, Center for Chemistry and Chemical Engineering, Lund University, \\ Box 124, SE-221 00, Lund, Sweden \\ Received November 26, 2001; Revised Manuscript Received March 25, 2002
}

\begin{abstract}
Intermolecular structures of complexes formed between a charged polymer and a spherical and oppositely charged macroion have been studied by employing the primitive model solved by Monte Carlo simulations. The strong-complex case involving a polyion and a macroion with equal absolute charges and without small ions was considered. The influence of the polyion flexibility with a bare persistence length ranging from 7 to $1000 \AA$ for four different systems characterized by different polyion linear charge densities and macroion sizes has been examined. Radial distributions, polyion bead complexation probabilities, loop, tail, and train characteristics, and energetic analysis have been performed. The strongest and most compact complex, involving a collapsed polyion wrapping the macroion, was formed for a semiflexible chain. As the stiffness was increased, this state was transformed into a range of different structures comprising "tennis ball seam"-like, solenoid, multiloop ("rosette"), and single-loop arrangements as well as structures involving only a single polyion-macroion contact region.
\end{abstract}

\section{Introduction}

Complex formation between charged polymers (polyions) and oppositely charged colloids (macroions) is of great interest both in practical applications and in theoretical investigations of modern colloidal science. In nature, polymers such as DNA, polyamines, many polysaccharides, etc., are charged, and many colloidal particles such as proteins, aggregates of them, liposomes, etc., are charged as well. The number of different manmade polyions is extensive, and in technological applications charged surfactant micelles, silica particles, latex particles, just to mention a few charged colloids, are widely used as well. The experimental studies in this area have a long history; see, e.g., the reviews by Goddard, ${ }^{1} \mathrm{~K}$ wak, ${ }^{2}$ Lindman and Thalberg, ${ }^{3}$ Hansson and Lindman, ${ }^{4}$ and Doublier et al. ${ }^{5}$ More recent studies $^{6-13}$ have also provided valuable insights.

During the past decade, the adsorption of polyions at oppositely charged surfaces has been subjected to an intense theoretical interest, and different theoretical approaches including simulation methods have been employed. Most of these studies involve a polyion adsorbing onto (i) planar surfaces representing monolayer and bilayer surfaces and surfaces of large particles, (ii) cylindrical surfaces representing e.g. histones, and (iii) spherical surfaces representing micelles, vesicles, globular proteins, dendrimeres, etc. Owing to the large number of reduced variables in these systems, much of the present theoretical developments do not yet overlap, even in the reduced parameter space. Hence, theories have not yet to any larger extent been confronted by essentially exact solutions of models provided by simulations.

The more recent theoretical investigations of the complexation between polyions and oppositely charged macroions are now extended,14-34 and some of the earlier studies have also recently been summarized. ${ }^{35}$ Most of these studies consider the complexation between one polyion and one macroion, but also the cases of

* Corresponding author. E-mail: Anna.Akinchina@fkem1.lu.se. several macroions ${ }^{27,31-34}$ and several polyions ${ }^{18}$ have been considered.

In more detail, Monte Carlo simulations have previously been employed to obtain structural and thermodynamical aspects of the polyion-macroion complexation. In a series of papers, ${ }^{15-17}$ Wallin and Linse examined such complexations applied to surfactant micelles at different conditions with the focus on thermodynamic aspects. Further simulations have been performed by Kong and Muthukumar ${ }^{19}$ and Nguyen and Shklovskiii 27 to test previous theoretical predictions. Chodanowski and Stoll have simulated the polyionmacroion complexation and examined how the complex structure depends on the macroion size ${ }^{28}$ and polyion chain length ${ }^{29}$ at different screening lengths. Moreover, $\mathrm{J}$ onsson and Linse have simulated the complexation between one polyion and varying amount of macroions, including the limit where the complex becomes saturated of macroions at different macroion and polyion extension and charge, 31 polyion stiffness, ${ }^{32}$ and salt content. ${ }^{34}$ In addition, the complexation between a polyion and a lysozyme has been simulated by Carlsson et al. ${ }^{30}$

The aim of the present study is to obtain further understanding on the complexation between a polyion and an oppositely charged macroion by performing systematic Monte Carlo simulations. We have employed a minimal system containing one polyion and one macroion with equal absolute charges and no small ions. Our focus is how the bare stiffness of the polyion affects the complex employing four different systems characterized by different polyion linear charge densities and sizes of macroion keeping the charges of the two components fixed. Despite the restricted selection of system parameters, a rich structural behavior is obtained. For flexible chains we obtain a collapsed polyion layer, as been considered in, e.g., refs 20, 22, 26, and 31 , whereas with stiffer chains "tennis ball seam"-like structure as previously observed by J eppesen et al., 36 multiloop or "rosette" structures as theoretically predicted by Schiessel et al., ${ }^{25}$ or solenoid arrangement as 
Table 1. Variables of the Systems Investigated

\begin{tabular}{|c|c|c|}
\hline variable & symbol & value \\
\hline \multicolumn{3}{|c|}{ General } \\
\hline volume & $\mathrm{V}$ & large \\
\hline temperature & $\mathrm{T}$ & $298 \mathrm{~K}$ \\
\hline relative permittivity & $\epsilon_{\mathrm{r}}$ & 78.5 \\
\hline Bjerrum length ${ }^{\mathrm{b}}$ & $\mathrm{L}_{\mathrm{B}} \equiv \mathrm{e}^{2} /\left(4 \pi \epsilon_{0} \epsilon_{\mathrm{r}} \mathrm{k} T\right)$ & $7.14 \AA$ \\
\hline \multicolumn{3}{|c|}{ Polyion } \\
\hline bead charge & $\mathrm{Z}_{\mathrm{b}}(>0)$ & 0.5 and $1^{c}$ \\
\hline bead radius & $\mathrm{R}_{\mathrm{b}}$ & $2.0 \AA$ \\
\hline bead-bead separation & b & $\approx 5.5 \AA$ \\
\hline no. of beads & $\mathrm{N}_{\mathrm{b}}$ & d \\
\hline bare persistence length & $I_{P}$ & $\begin{array}{l}\text { 7, } 30,60,120,250 \text {, } \\
500 \text {, and } 1000 \AA\end{array}$ \\
\hline linear charge densityb & \multicolumn{2}{|l|}{$\lambda_{\mathrm{p}} \equiv \mathrm{e}_{\mathrm{b}} / \mathrm{b}$} \\
\hline polyion contour length ${ }^{b}$ & \multicolumn{2}{|l|}{$L \equiv b\left(N_{b}-1\right)$} \\
\hline \multicolumn{3}{|c|}{ Macroion } \\
\hline charge & $\mathrm{Z}_{\mathrm{m}}(<0)$ & -40 \\
\hline radius & $\mathrm{R}_{\mathrm{m}}$ & 10 and $20 \AA^{c}$ \\
\hline surface charge density ${ }^{b}$ & \multicolumn{2}{|l|}{$\sigma_{\mathrm{m}} \equiv \mathrm{e}_{\mathrm{m}} /\left(4 \pi \mathrm{R}_{\mathrm{m}}{ }^{2}\right)$} \\
\hline \multicolumn{3}{|c|}{ Small Ions } \\
\hline $\begin{array}{l}\text { salt number density } \\
\text { ion charges } \\
\text { ion radii }\end{array}$ & $\begin{array}{l}\rho \mathrm{S} \\
\mathrm{Z}_{+}, \mathrm{Z}_{-} \\
\mathrm{R}_{+}, \mathrm{R}_{-}\end{array}$ & 0 \\
\hline
\end{tabular}

a $A$ spherical cell with radius $R_{\text {cell }}=1241 \AA$ was used. The precise value of the volume is in practice irrelevant, owing to the strong complexation free energy. For example, the polyion mixing entropy becomes relevant first when the system volume $\mathrm{V} \approx$ $V_{\text {complex }} \exp \left(\mathrm{A}_{\text {complex }} / \mathrm{kT}\right) \approx 10^{15} \mathrm{~m}^{3}$, estimated by using a complexation volume $V_{\text {complex }}=10^{2} \AA^{3}$ and a complexation free energy $\mathrm{A}_{\text {complex }} / \mathrm{kT}=100 .{ }^{\mathrm{b}}$ Dependent variable. ${ }^{\mathrm{c}}$ See Table 2. ${ }^{\mathrm{d}}$ Fixed by the requirement $Z_{p} \equiv N_{b} Z_{b}=-Z_{m}$.

assumed by Schiessel et al. ${ }^{33}$ and by Nguyen and Shklovskii ${ }^{26}$ are observed. Our work is also related to the theoretical predictions by Netz and J oanny, ${ }^{23}$ where they investigated the "touching" and "wrapping" transitions, and it also complements the recent simulation studies by Chodanowski and Stoll. 28,29

The paper is organized as follows: Section II describes the model system and some simulation aspects. Our results are presented in section III and discussed in section IV. In section V, we conclude our findings, and the paper ends with an appendix, where we compare and discuss different methods to calculate the persistence length of a polymer.

\section{Method}

2.1. Model. The complex formed by one polyion and one macroion is described in the framework of the primitive model and using a cell model. The former implies that (i) the charged species (charged polyion segments and macroions) are represented as charged hard spheres, each specified by a charge and a hardsphere radius, and (ii) the solvent is treated as a continuum with a constant relative permittivity. The latter involves a confinement of the species inside a spherical cell, and in the present study the macroion was also fixed at the center of the cell.

As alluded to in the Introduction, the present system is described by a large number of parameters. To clarify the situation and to make comparison with other studies more transparent, we will provide a relevant set of variables and motivate our choice of systems investigated.

A system comprised of one polyion and one macroion with their counterions and additional salt of the same type as the counterions described within the primitive model requires 15 physical rel evant variables. Table 1
Table 2. Specific Variables and Some Results of the Systems Investigated

\begin{tabular}{|c|c|c|c|c|}
\hline system & I & II & III & IV \\
\hline \multicolumn{5}{|c|}{ Independent Variables } \\
\hline $\begin{array}{l}Z_{b} \\
R_{m}(\AA)\end{array}$ & $\begin{array}{l}0.5 \\
20\end{array}$ & $\begin{array}{l}0.5 \\
10\end{array}$ & $\begin{array}{l}1 \\
20\end{array}$ & $\begin{array}{l}1 \\
10\end{array}$ \\
\hline \multicolumn{5}{|c|}{ Important Dependent Variables } \\
\hline $\mathrm{N}_{\mathrm{b}}$ & 80 & 80 & 40 & 40 \\
\hline$\Gamma_{\mathrm{mb}} \equiv\left|\mathrm{Z}_{\mathrm{m}} \mathrm{Z}_{\mathrm{b}}\right| \mathrm{L}_{\mathrm{B}} /\left(\mathrm{R}_{\mathrm{m}}+\mathrm{R}_{\mathrm{b}}\right)$ & 6.50 & 11.9 & 13.0 & 23.8 \\
\hline contour length $L=b\left(N_{b}-1\right)(\AA)^{a}$ & 430 & 430 & 210 & 210 \\
\hline$L /\left[2 \pi\left(R_{b}+R_{m}\right)\right]$ & 3.1 & 5.8 & 1.6 & 2.8 \\
\hline bead coverage $N_{b}\left(\pi R_{b}^{2}\right) /\left(4 \pi R_{m}^{2}\right)$ & 0.2 & 0.8 & 0.1 & 0.4 \\
\hline $\begin{array}{l}\text { bond coverage } \\
\qquad N_{b}\left(\pi(b / 2)^{2}\right) /\left(4 \pi \mathrm{R}_{m}^{2}\right)^{\mathrm{b}}\end{array}$ & 0.4 & 1.5 & 0.2 & 0.7 \\
\hline \multicolumn{5}{|c|}{ Bead-Bead Separation } \\
\hline$\left\langle\mathrm{R}_{\mathrm{bb}}^{2}\right\rangle^{1 / 2}(\AA)\left(\mathrm{I}_{\mathrm{P}}=7 \AA\right)$ & 5.68 & 5.55 & 5.91 & 5.64 \\
\hline$\left\langle R_{\mathrm{bb}}^{2}\right\rangle^{1 / 2}(\AA)\left(I_{\mathrm{p}}=60 \AA\right)$ & 5.54 & 5.50 & 5.73 & 5.29 \\
\hline$\left.\left\langle\mathrm{R}_{\mathrm{bb}}^{2}\right\rangle\right\rangle^{1 / 2}(\AA)\left(\mathrm{I}_{\mathrm{P}}=1000 \AA\right)$ & 5.53 & 5.47 & 5.39 & 5.23 \\
\hline
\end{tabular}

displays one such set of variables, their symbols, numerical values used, and some other variables derived from these. In the present study, we have focused on systems (i) in which the polyion and the macroion carry same absolute charges, (ii) free of small ions, and (iii) where the cell radius $R_{\text {cell }}$ is sufficiently large to make the volume in practice irrelevant, thus leaving eight variables left. Moreover, the focus on (iv) aqueous solution at ambient temperature fixes $T$ and $\epsilon_{r}$ and ( $v$ ) alkyl-based polyions restricts the bead-bead separation and bead size to a narrow window. Finally, only strong complexes formed are of interest, and the macroion charge $Z_{m}=\left(-Z_{p}=\right)-40$ has been assigned throughout.

The three remaining independent variables are (i) the macroion radius $R_{m}$, (ii) the bead charge $Z_{b}$, and (iii) the chain flexibility here expressed by the bare persistence length $I_{p}$. Table 2 specifies the four different systems considered, which are obtained by combining the bead charges 0.5 and 1 with the macroi on radi 10 and $20 \AA$. The properties of these four systems have been investigated at seven different chain flexibilities, ranging from fully flexible chains, $I_{P}=7 \AA$, to nearly rigid rods, $I_{p}=1000 \AA$ (see al so Table 1 ). As presented below, the variation of these three parameters is sufficient to monitor a rich behavior of the structure of the polyion-macroion complex.

The polyion is represented by a chain of charged beads connected by harmonic bonds. The total potential energy for a system can be expressed as

$$
\mathrm{U}=\mathrm{U}_{\mathrm{hc}}+\mathrm{U}_{\mathrm{el}}+\mathrm{U}_{\text {bond }}+\mathrm{U}_{\text {angle }}
$$

where the terms describing the hard-core repulsion and the Coulomb interaction are given by

$$
\begin{gathered}
U_{h c}+U_{e l}=\sum_{i<j} U_{i j} \\
U_{i j}= \begin{cases}\infty & r_{i j}<R_{i}+R_{j} \\
\frac{Z_{i} Z_{j} e^{2}}{4 \pi \epsilon_{0} \epsilon_{r} r_{i j}} & r_{i j} \geq R_{i}+R_{j}\end{cases}
\end{gathered}
$$

with $Z_{i}$ and $R_{i}$ denoting the charge and the radius of particle i (the macroion or a polyion bead), e the elementary charge, $\epsilon_{0}$ the permittivity of vacuum, $\epsilon_{\mathrm{r}}$ the relative permittivity of water, and $r_{i j}$ the distance between the centers of particles $i$ and $j$. 
The polymer chain connectivity and flexibility are controlled by the bond and angular harmonic potential energies according to

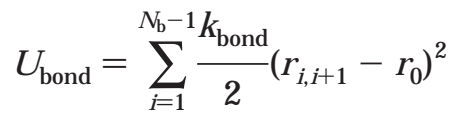

and

$$
\mathrm{U}_{\text {angle }}=\sum_{\mathrm{i}=2}^{\mathrm{N}_{\mathrm{b}}-1} \frac{\mathrm{k}_{\text {angle }}}{2}\left(\alpha_{\mathrm{i}}-\alpha_{0}\right)^{2}
$$

respectively, where $\mathrm{N}_{b}$ is the number of beads in the polyion, $\mathrm{k}_{\text {bond }}$ the bond force constant, $\mathrm{r}_{\mathrm{i}, \mathrm{i}+1}$ the distance between two connected beads, $r_{0}$ the zero-force bond distance, $k_{\text {angle }}$ the angle force constant, $\alpha_{i}$ the angle between three consecutive beads (bond angle), and $\alpha_{0}$ the zero-force bond angle. Here, we have used $r_{0}=5.0$ $\AA$ and $\mathrm{k}_{\text {bond }}=0.4 \mathrm{~N} / \mathrm{m}$, which in combination with the reaming interactions leads to a root-mean-square ( $\mathrm{rms}$ ) bead-bead separation $\left\langle\mathrm{R}_{\mathrm{bb}}^{2}\right)^{1 / 2} \approx 5.5 \AA$. Moreover, $\alpha_{0}=$ $180^{\circ}$ was selected, and $k_{\text {angle }}=0,3.6,7.7,15.8,33.5$, 67.4 , and $135 \mathrm{~J} /(\mathrm{mol} \mathrm{deg})$ were used, which give the bare persistence lengths (the persistence length calculated for an uncharged chain) displayed in Table 1 . The persistence length $I_{p}$ was evaluated according to

$$
I_{P}=\frac{\left\langle R_{b b}^{2}\right\rangle^{1 / 2}}{1+\left\langle\cos \alpha_{i}\right\rangle}
$$

and a more detailed description of its evaluation is presented in the Appendix. The chains of different stiffness will be labeled according to their bare persistence lengths.

The strong electrostatic interaction between the polyion and the macroion gives rise to a firm complex at all conditions. A central quantity is the reduced el ectrostatic interaction of a polyion bead at hard-sphere contact with the macroion, $\Gamma_{m b} \equiv\left|Z_{m} Z_{b}\right| L_{B} /\left(R_{m}+R_{b}\right)$, where $L_{B}$ is the Bjerrum length (see Table 1 ). The weakest interaction appears in system I (characterized by $Z_{b}=0.5, N_{b}=80$, and $R_{m}=20 \AA$ ) where $\Gamma_{m b}=6.50$. In systems II $\left(Z_{b}=0.5, N_{b}=80\right.$, and $\left.R_{m}=10 \AA\right)$ and III $\left(Z_{b}=1, N_{b}=40\right.$, and $\left.R_{m}=20 \AA\right)$, we have $\Gamma_{m b}=$ 11.9 and 13.0, respectively, and the strongest interaction appears in system IV $\left(Z_{b}=1, N_{b}=40\right.$, and $\left.R_{m}=10 \AA\right)$ where $\Gamma_{\mathrm{mb}}=23.8$. These and geometrical data as the polyion contour length, the polyion length divided by the circumference of the macroion, and the bead and the bond coverage are collected in Table 2.

2.2. Simulation Details. All Monte Carlo (MC) simulations were performed in the canonical ensemble, employing the standard Metropolis algorithm. ${ }^{37}$ Since the position of the macroion was fixed, only the polyion beads were subjected to trial moves. F our different types of MC trial moves were employed: (i) single bead move, (ii) pivot rotation where the chain is randomly divided into two parts and the shortest of them rotated around the point of division, (iii) translation of the entire chain, and (iv) slithering move where one of the end beads is moved to the opposite end of the chain with biased radial and angular positioning. The single particle move was attempted 50 times more often than the other three types of moves. The displacement parameters of the different types of trial moves were selected for each case within the following ranges: $\Delta_{\text {bead }}=0.7-2.5 \AA, \Delta_{\alpha}=$ $15^{\circ}-360^{\circ}$, and $\Delta_{\text {chain }}=0.3-0.6 \AA$. The average acceptance probabilities of the four types of moves were $\approx 0.4, \approx 0.4, \approx 0.5$, and $\approx 0.6$.

Most often the polymer beads were initially placed randomly in the simulation cell and separated from the macroion fixed at the center, leading to separated macromolecules. The simulation of each complex involved typically an equilibration of $5 \times 10^{5} \mathrm{MC}$ passes (trial moves per particle) followed by a production run of $1 \times 10^{6} \mathrm{MC}$ passes. In cases with large bare persistence lengths, it was expected that the equilibrium process could be trapped in metastable conformations characterized by the number of polyion loop (cf. Figure $1)$. Therefore, systems I and II with $I_{P} \geq 250 \AA$ and system IV with $I_{p}=1000 \AA$ were simulated with different initial configurations. In addition to separated macromolecules, al so collapsed complexes achieved from simulations with shorter persistence lengths were employed as initial configurations. In those cases where different initial configurations produced states characterized by different number of loops (system I with I $=$ $1000 \AA$ and system II with $I_{P}=500$ and $1000 \AA$ ), results of the state with the lowest potential energy of each system are reported, assuming the entropy differences among the states being less important.

Reported errors are one standard deviation, and they are evaluated by diving the total simulation into typical 10 subbatches. All the simulations were carried out with the use of the integrated Monte Carlo/molecular dynamics/Brownian dynamics simulation package MOLSIM. 38

\section{Results}

3.1. Overview. An overview of the structures of the complexes obtained is given in Figure 1. The final configurations of the simulations are displayed for systems I-IV (from left to right) with five of the seven flexibilities consider ed (increasing $I_{p}$ from top to bottom).

These configurations illustrate that a polyion-macroion complex appears at all conditions, which also is confirmed by the statistical analysis presented below. Another general observation is that with a fully flexible chain (top row) all polyion beads are near the macroion in a disordered manner. Furthermore, as the chain stiffness is increased progressively, the chain becomes locally less folded, and configurations such as a "rosette" (system II, IP $=250 \AA$ ), a "tennis ball seam" (system III , IP $=60 \AA$ ), and a solenoid (system IV , Ip $=60-500$ $\AA$ ) occur. With the largest stiffness, $I_{p}=1000 \AA$ (bottom row), only a limited number of polyion beads appear near the macroion, and depending on the system a single loop (systems I, II, and IV) or a U-turn (system III) occurs. Moreover, in systems I and II with $\mathrm{I}_{\mathrm{P}}=1000$ $\AA$, metastable conformations (see section 2.2) with a slightly to moderately bent polyion making only a single contact with the macroion were also obtained. Here, the macroion was in contact with only a few central beads forming a bend of the chain.

Hence, the four systems employed, being composed of one polyion and one macroion with same absolute charges, display a rich sequence of structures as the bare persistence length is altered, and moreover the sequences of structures differ among the systems.

3.2. Complex Structure. 3.2.1. Radial Bead Distribution. The number of beads within the distance $r$ from the center of the macroion will now be considered. The so-called running coordination number, $r \mathrm{cn}(r)$, has been 


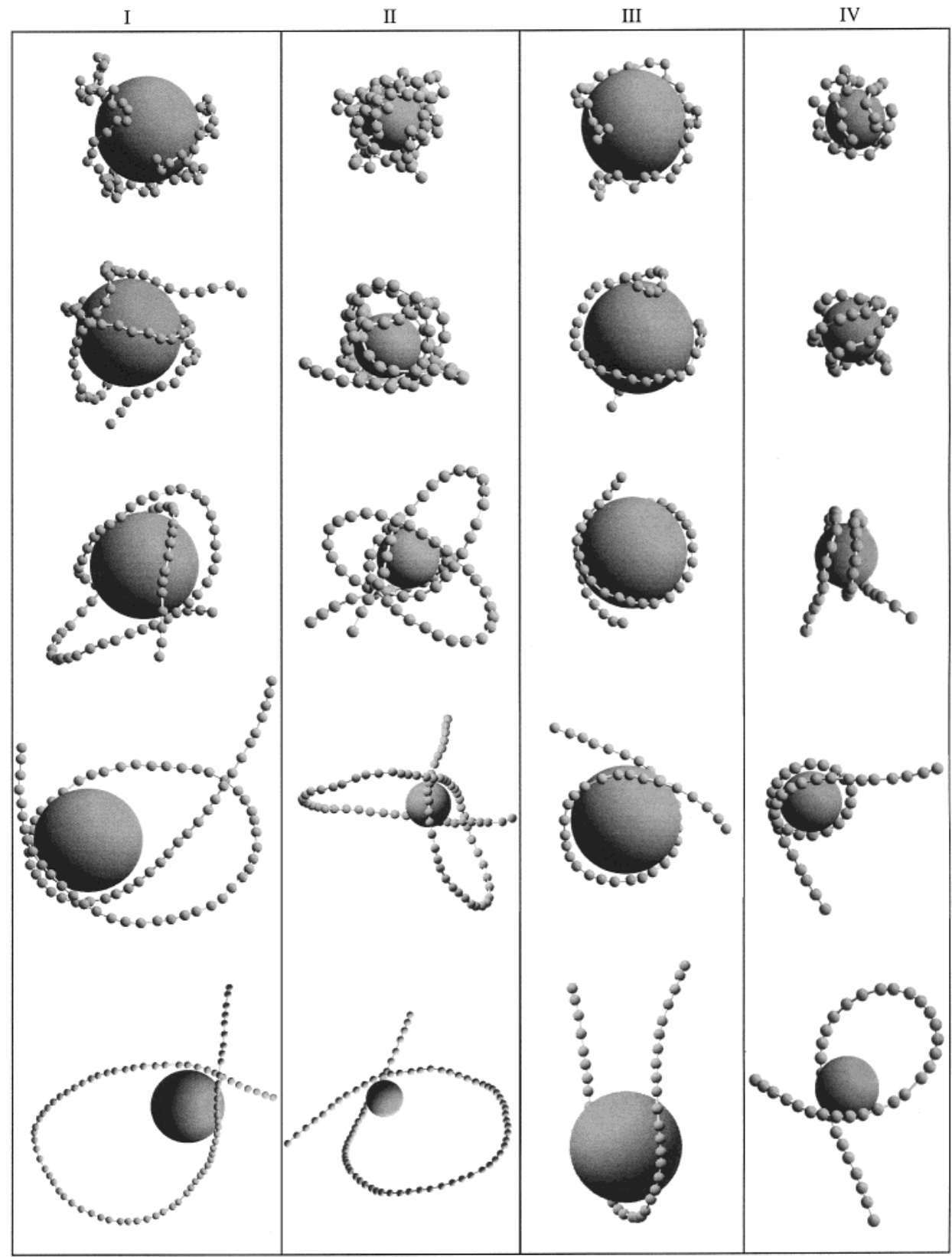

Figure 1. Final configurations from the simulations of systems I-IV (from left to right) with bare persistence lengths $I_{P}=7,60$, 250,500 , and $1000 \AA$ (from top to bottom). The same length scale is used throughout except for systems I with IP $=1000 \AA$ and system II with $I_{P}=500$ and $1000 \AA$.

calculated, and the function will furthermore be normalized by the number of polyion beads $\mathrm{N}_{\mathrm{b}}$. Hence, the normalized running coordination number, $\mathrm{rcn}(\mathrm{r}) / \mathrm{N}_{\mathrm{b}}$, expresses the fraction of beads located within the distance $r$ from the center of the macroion, and by definition $\mathrm{rcn}(\mathrm{r}) / \mathrm{N}_{\mathrm{b}}$ is zero for $\mathrm{r}<\mathrm{R}_{\mathrm{m}}+\mathrm{R}_{\mathrm{b}}$ and approaches unity at large $r$.

Figure 2 displays the normalized $r c n(r)$ for each of the four systems with chains of different bare persistence lengths. Generally, all functions display a sharp increase at the macroion-bead contact separation $r=$ $R_{m}+R_{b}$ and thereafter a leveling off, although the steepness of the initial rise and the distance at which the limiting value is approached differ among the systems.

In more detail, the initial increase of the reduced $r c n$ is less steep in systems I and II $\left(Z_{b}=0.5\right)$ as compared to those in systems III and IV $\left(Z_{b}=1\right)$. With the completely flexible chains $\left(I_{P}=7 \AA\right)$, nearly all beads are within $r=R_{m}+R_{b}+10 \AA$ in the two former systems, whereas in the two latter systems a $5 \AA$ thick layer is sufficient. This difference in the thickness of the polyion layer at the macroi on surface is also visible in Figure 1.

Moreover, as the stiffness is slightly increased, the steepness of the reduced rcn increases in systems I, III, and IV. Apparently, the accumulation of the oppositely charged polyion beads is facilitated by some rigidity of the chain, an observation that we will return to. In contrast, in system II the steepness of the increase of the reduced rcn becomes smaller, and the approach to the limiting value appears at larger distances as the chain stiffness is increased-features that are consistent with the appearance of beads at larger separation from the macroion with increasing $I_{p}$ displayed in Figure 1. 

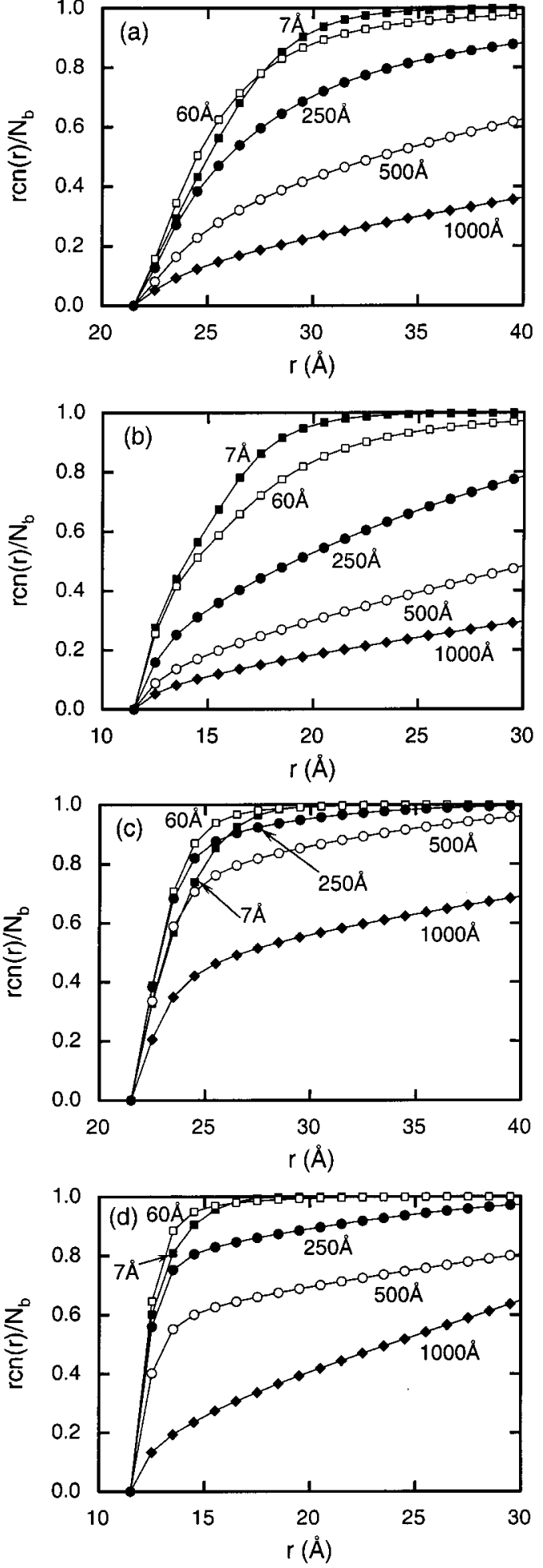

Figure 2. Normalized running coordination number $[\mathrm{rcn}(\mathrm{r}) /$ $\mathrm{N}_{\mathrm{b}}$ ] as a function of the distance from the center of the macroion ( $r$ ) for systems (a) I , (b) II, (c) III, and (d) IV for indicated bare persistence lengths. Estimated errors are smaller than the size of the symbols.

3.2.2. Bead Complexation Probability. The configurations of the polyion will now be examined more thoroughly. The division of the beads into two groups, collapsed and noncollapsed, is frequently used in theory, and this division is employed in our analysis as well. Here, we adopt a geometrical definition and consider a bead as being collapsed if it is located within $5 \AA$ from the closest approach of the macroion surface, i.e., when the separation between the centers of a bead and of the macroion does not exceed the distance $r=R_{c} \equiv R_{m}+$ $R_{b}+5 \AA$. As obvious from Figure 2, there is some ambiguity of selecting $R_{c}$; however, the qualitative conclusions are not affected by the precise choice.

The complexation probability function, $\mathrm{P}_{\mathrm{c}}\left(\mathrm{i}_{\mathrm{b}}\right)$, providing the probability that bead $i_{b}$ of the chain being complexed with the macroion, will now be employed to obtain detailed information on the structure of the complex. The function possesses the lower boundary $P_{c}\left(i_{b}\right)=0$, implying that bead $i_{b}$ is never complexed with the macroi on, and the upper one $\mathrm{P}_{\mathrm{c}}\left(\mathrm{i}_{\mathrm{b}}\right)=1$, corresponding to the case that this bead is al ways complexed. Such analyses have previously provided valuable information in studies ${ }^{30-32}$ on different systems containing polyions and charged spherical colloids.

Figure 3 shows the complexation probabilities as a function of $\mathrm{i}_{\mathrm{b}}$ for the cases displayed in Figure 1. I deally, these probabilities should be symmetric with respect to a reflection at $\mathrm{i}_{\mathrm{b}}=\left(\mathrm{N}_{\mathrm{b}}+1\right) / 2$. This is fulfilled to a very high degree, and the use of the biased slithering technique to generate trial moves was in practice necessary for such an achievement.

Starting with system I, Figure 3a displays how $P_{c}\left(i_{b}\right)$ evolves with increasing chain stiffness. For the fully flexible chain $\left(I_{p}=7 \AA\right)$, the complexation function displays an extended plateau at $\approx 0.7$ and a reduced probability of complexation toward the ends of the chain. Such a plateau or several sections with high complexation probability will be referred to as binding region(s). With increasing chain stiffness $\left(I_{p}=60 \AA\right)$, the probability of a bead in the binding region to be complexed increases and the tails becomes more pronounced. With an even larger bare persistence length $\left(I_{p}=250 \AA\right)$, three maxima appear signaling the appearance of two loops, and with $I_{P}=500$ and $1000 \AA$ two well-separated maxima occur revealing one extended loop. All these findings are consistent with the configurations shown in Figure 1.

Figure $3 b$ provides the corresponding complexation functions for system II comprising a smaller macroion, $\mathrm{R}_{\mathrm{m}}=10 \AA$, as compared to system I. Here, we observe a similar behavior with increasing stiffness as in system I, i.e., extended binding region for flexible chains and the appearance of loops at increasing stiffness. However, three clear differences do also appear, viz. (i) in the central region $P_{C}\left(i_{b}\right)$ decreases continuously as $I_{P}$ is increased, (ii) four maxima appear $\left(I_{p}=250 \AA\right)$, indicating the appearance of three loops, and (iii) three pronounced maxima occur $\left(I_{P}=500 \AA\right)$, implying the presence of two well-defined loops, all the differences consistent with the snapshots in Figure 1.

Regarding system III, the linear charge density of the polyion is twice as large and the length of the polyion half as long as in system I. The corresponding complexation functions for system III are given in Figure 3c. With the fully flexible chain, we again observe an extended binding region and short tails, here the probability of a bead in the binding region to be complexed being $\approx 0.95$. As the chain becomes stiffer, (i) the complexation probability increases (as in system I), but remains near one even with the stiffest chain, and (ii) the tail becomes progressively longer. Obviously, the central part of the chain is always near the macroion surface, and no loops do appear. With the stiffest chain, the U-turn is composed of ca. 20 beads. 

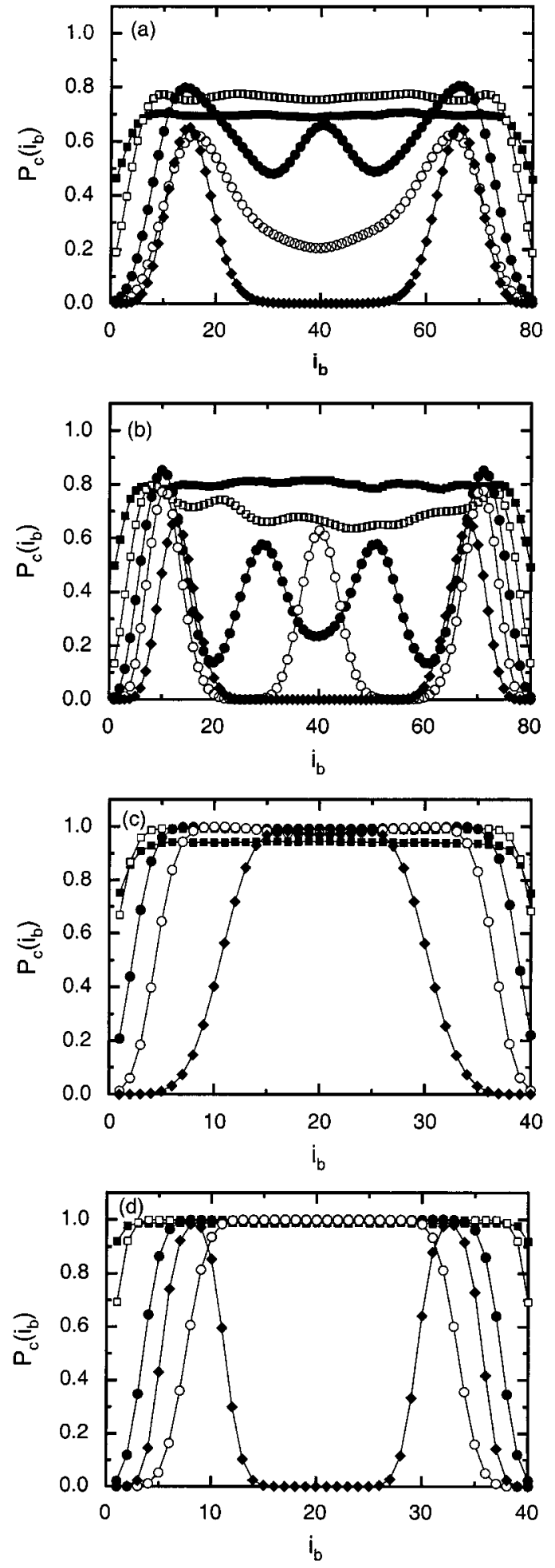

Figure 3. Complexation probability $\left[\mathrm{P}_{\mathrm{c}}\left(\mathrm{i}_{\mathrm{b}}\right)\right]$ as a function of the bead number $\left(\mathrm{i}_{\mathrm{b}}\right.$ ) for systems (a) I, (b) II , (c) III , and (d) IV with bare persistence lengths $I_{p}=7 \AA$ (filled squares), $60 \AA$ (open squares), $250 \AA$ (filled circles), $500 \AA$ (open circles), and $1000 \AA$ (filled diamonds). Estimated errors are smaller than the size of the symbols, except for system II with $I_{p} \geq 60 \AA$ where they are 0.05-0.09.

Finally, the corresponding data for system IV are given in Figure $3 \mathrm{~d}$. Throughout, most of the complexed beads display a complexation probability near one. With the completely flexible chain, essentially all beads are collapsed $\left[\mathrm{P}_{\mathrm{c}}\left(\mathrm{i}_{\mathrm{b}}\right) \approx 1\right.$ for all $\left.\mathrm{i}_{\mathrm{b}}\right]$, and with $\mathrm{I}_{\mathrm{P}}=60 \AA$ only the end beads display a slight probability of being detached. With an increased stiffness, the tails becomes progressively longer. First with $I_{p}=1000 \AA$, do a single loop appear, but even in this case considerable tails are present. The shortening of the binding region at increasing $I_{p}$ up to $I_{p}=500 \AA$ is consistent with the progressively unwrapping of the solenoid displayed in Figure 1.

Thus, the complexation probabilities provide a fingerprint of the chain configurations and confirm that the configurations shown in Figure 1 indeed represent typical structures of the systems. We al so conclude that in the present systems with equal absolute charge of the macromolecules the macroion prefer to complex to the central part rather than to one end of the polyion (symmetric complexation).

3.2.3. Loop, Tail, and Train Characteristics. The chain conformations have furthermore been characterized by quantifying (i) the average number of beads in a loop, tail, and train, (ii) the average number of loops, tails, and trains, and (iii) the fraction of beads in loops, tails, and trains. Here, train refers to a contiguous sequence of beads at the macroion surface, and in this analysis we have used the same definition of $R_{c}$ as above. Figure 4 displays the number of beads in a feature of type $\alpha$, $\left\langle\mathrm{N}_{\mathrm{b}}\right\rangle_{\alpha}, \alpha=$ (loop, tail, or train), as a function of the bare persistence length for the four systems, whereas the insets of Figure 4 show the corresponding average number of feature $\alpha,\left\langle\mathrm{N}_{\alpha}\right\rangle$ (except for $\left\langle\mathrm{N}_{\text {train }}\right\rangle$, since $\left\langle\mathrm{N}_{\text {train }}\right\rangle$ $\left.\equiv\left\langle\mathrm{N}_{\text {loop }}\right\rangle+1\right)$. From $\left\langle\mathrm{N}_{\mathrm{b}}\right\rangle_{\alpha}$ and $\left\langle\mathrm{N}_{\alpha}\right\rangle$, the fraction of beads in feature $\alpha$ is readily available through $\mathrm{P}_{\alpha}=\left\langle\mathrm{N}_{\mathrm{b}}\right\rangle_{\alpha}\left\langle\mathrm{N}_{\alpha}\right\rangle /$ $\mathrm{N}_{\mathrm{b}}$.

Regarding systems I and II, Figure 4a quantifies the appearance of numerous short loops extending from the collapsed layer with $I_{p}=7 \AA$, and the successive reduction of number of loops and the enlargement of the loops as the bare persistence length is increased. The fraction of beads being in a loop, $\mathrm{P}_{\text {loop, }}$ increases monotonically and displays a maximum for the stiffest chains (e.g., $P_{\text {loop }}=0.62$ for system II with $I_{p}=1000$ $\AA$ ). Systems III and IV also display a reduction of the number of loops with increasing $I_{p}$, but $\left\langle\mathrm{N}_{\text {loop }}\right\rangle$ is small already for flexible chains. The length of the loops appearing is short, just one or two beads. Hence, also the fraction of beads in loops is small. Regarding system $\mathrm{IV}$, the length of the singl e loop appearing at $I_{\mathrm{p}}=1000$ $\AA$ is nearly 20 beads (half the chain).

Figure $4 \mathrm{~b}$ shows that the number of tails and the average tail length, and hence also the fraction of beads in tails, generally increase as the chain stiffness is increased. Moreover, the four systems can also be divided into the same two groups according to how the tail statistics depends on the bare persistence length. In systems I and II, $\left\langle\mathrm{N}_{\mathrm{b}}\right\rangle_{\text {tail }}$ and $\left\langle\mathrm{N}_{\text {tail }}\right\rangle$ are larger than those in systems III and IV. With flexible chains, systems I and II possess chains with on the average tails containing ca. 3 beads, whereas in systems III and IV such tails lengths appears first at $I_{p} \approx 250 \AA$. As the chain stiffness increases, $\left\langle\mathrm{N}_{\mathrm{b}}\right\rangle_{\text {tail }}$ in systems II and III increases monotonically, displaying the maximum for the stiffest chains. In systems I and IV, $\left\langle\mathrm{N}_{\mathrm{b}}\right\rangle_{\text {tail }}$ shows a nonmonotonic behavior with the maximum at $\mathrm{I}_{\mathrm{p}} \approx 500$ $\AA$ and decay for the stiffest chains considered. Finally, we notice that we generally do not have two tails. With the exception of system III, first with $I_{p} \geq 250 \AA$ we find $\left\langle\mathrm{N}_{\text {taii }}\right\rangle>1.95$, demonstrating the nearly full development of two tails. 

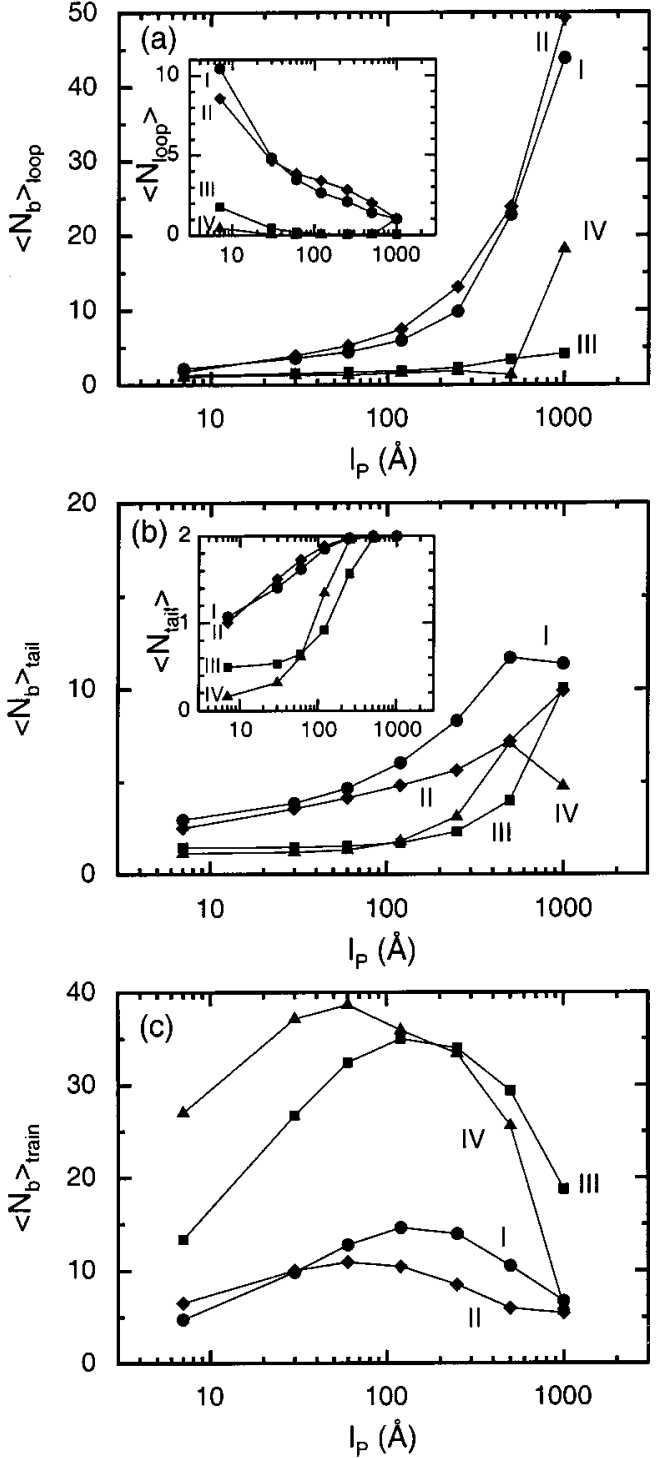

Figure 4. Number of beads in (a) a loop $\left(\left\langle\mathrm{N}_{\mathrm{b}}\right\rangle_{\text {loop }}\right)$, (b) a tail $\left(\left\langle\mathrm{N}_{\mathrm{b}}\right\rangle_{\text {tail }}\right)$, and (c) a train $\left(\left\langle\mathrm{N}_{\mathrm{b}}\right\rangle_{\text {train }}\right)$ as a function of the bare persistence length $\left(I_{P}\right)$ for indicated systems. The inserts in (a) and (b) display the number of loops $\left\langle\mathrm{N}_{\text {loop }}\right\rangle$ and tails $\left\langle\mathrm{N}_{\text {tail }}\right\rangle$, respectively. The lines are only guide for the eye.

The number of beads in a train is given in Figure 4c. The general behavior is that $\left\langle\mathrm{N}_{\mathrm{b}}\right\rangle_{\text {train }}$ displays a maximum at $I_{p} \approx 100 \AA$; i.e., the longest contiguous sequence of collapsed beads appears at intermediate stiffness. Also, here a 2-fold change in the macroion radius (cf. curves label ed I and II) has a smaller effect than a 2-fold change in the linear charge density (cf. curves I and III). Systems I and II containing the longer chains but with lower linear charge density display a smaller number of beads in a train as compared to systems III and IV.

A comparison between the data given in Figure 4 and the snapshots in Figure 1 reveals again a consistency between the statistical analysis and the complexes shown.

3.3. Degree of Charge Neutralization. A central quantity in polyion-macroion complexation is the ratio of charges carried by the polyion beads in the collapsed layer and the macroion charge denoted by $\left|Z_{\mathrm{p}}^{\mathrm{c}} / \mathrm{Z}_{\mathrm{m}}\right|$. This quantity is often taken as a measure of the strength of the polyion-macroion complex. In our system with a 1:1 complex and $\left|Z_{p} / Z_{m}\right|=1$, the upper limit of $\left|Z_{p}^{c} / Z_{m}\right|$

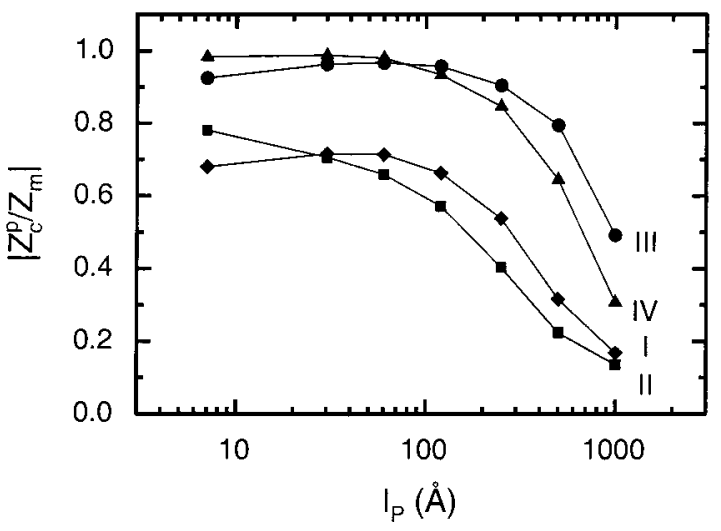

Figure 5. Charge ratio $\left(\left|Z_{p}^{c} / Z_{m}\right|\right)$ as a function of the bare persistence length $\left(I_{P}\right)$ for indicated systems. The lines are only guide for the eye. Estimated errors are smaller than the size of the symbols.

becomes unity, and the charge ratio is also equivalent to the fraction of beads in the collapsed layer.

Figure 5 shows the charge ratio $\left|Z_{p}^{c} / Z_{m}\right|$ as a function of the bare persistence length for the four systems. In systems I, III, and IV, the charge ratio displays an initial rise, a maximum, and then a decrease as the stiffness is increased, whereas in system $I,\left|Z_{p}^{c} / Z_{m}\right|$ is a continuously decaying function. The maxima of the charge ratio appearing in systems I, III, and IV are of course related to the nonmonotonic rise of the reduced rcn with increasing $I_{P}$ (Figure 2 ) and the nonmonotonic value of $P_{C}\left(i_{b}\right)$ in the binding region with increasing $I_{P}$ (Figure 3). The maxima in $\left|Z_{\mathrm{p}}^{\mathrm{C}} / Z_{\mathrm{m}}\right|$ appear at $I_{\mathrm{p}}=45$, 65 , and $30 \AA$ (from harmonic fits), respectively. In systems III and IV, $\left|Z_{p}^{\mathrm{C}} / Z_{\mathrm{m}}\right|$ approaches nearly unity at its optimal I expressing a nearly complete charge neutralization, whereas in systems I and II the maximal charge compensation approaches ca. 70 and $80 \%$, respectively; thus, the smaller bead charge makes the complex weaker. System IV with the largest $\Gamma_{\mathrm{mb}}$ displays a slightly larger maximum than system III, but the degree of neutralization becomes the opposite at larger chain stiffness. Obviously, with flexible chains the stronger macroion-bead attraction occurring with the smaller macroion in system IV facilitates the charge neutralization, whereas with stiffer chains, the larger electrostatic interaction does not compensate the energetic bending penalty of having the chain to follow the surface with the smaller curvature. In system III, even at $I_{p}=1000 \AA$, ca. $50 \%$ of the beads remain in the collapsed layer, consistent with the finding in section 3.2.2 of having ca. 20 beads in the U-turn.

3.4. Energetics. Additional insight in the driving force of the complexation and structures attained is obtained by examining the contributions to the potential energy of the systems. Figure 6 displays the average angular, electrostatic macroion-bead, and el ectrostatic bead-bead potential energy as a function of the bare persistence length for the four systems. In addition, corresponding electrostatic potential energies for two simple models are given in Figure 6 (open symbols). In the first model (the collapsed model), all beads are dissociated and placed in hard-sphere contact with the macroion. Then, we have $\mathrm{U}_{\mathrm{mb}} / \mathrm{N}_{\mathrm{b}} \mathrm{kT}=\Gamma_{\mathrm{mb}}$, whereas $\mathrm{U}_{\mathrm{bb}} /$ $\mathrm{N}_{\mathrm{b}} \mathrm{kT}$ was determined by numerical minimization of the total potential energy. In fact, $\mathrm{U}_{\mathrm{bb}} / \mathrm{N}_{\mathrm{b}} \mathrm{kT}$ does not deviate more than $20 \%$ from $-\Gamma_{\mathrm{mb}} / 2$ being the bead-bead energy of having the bead charges homogeneously 

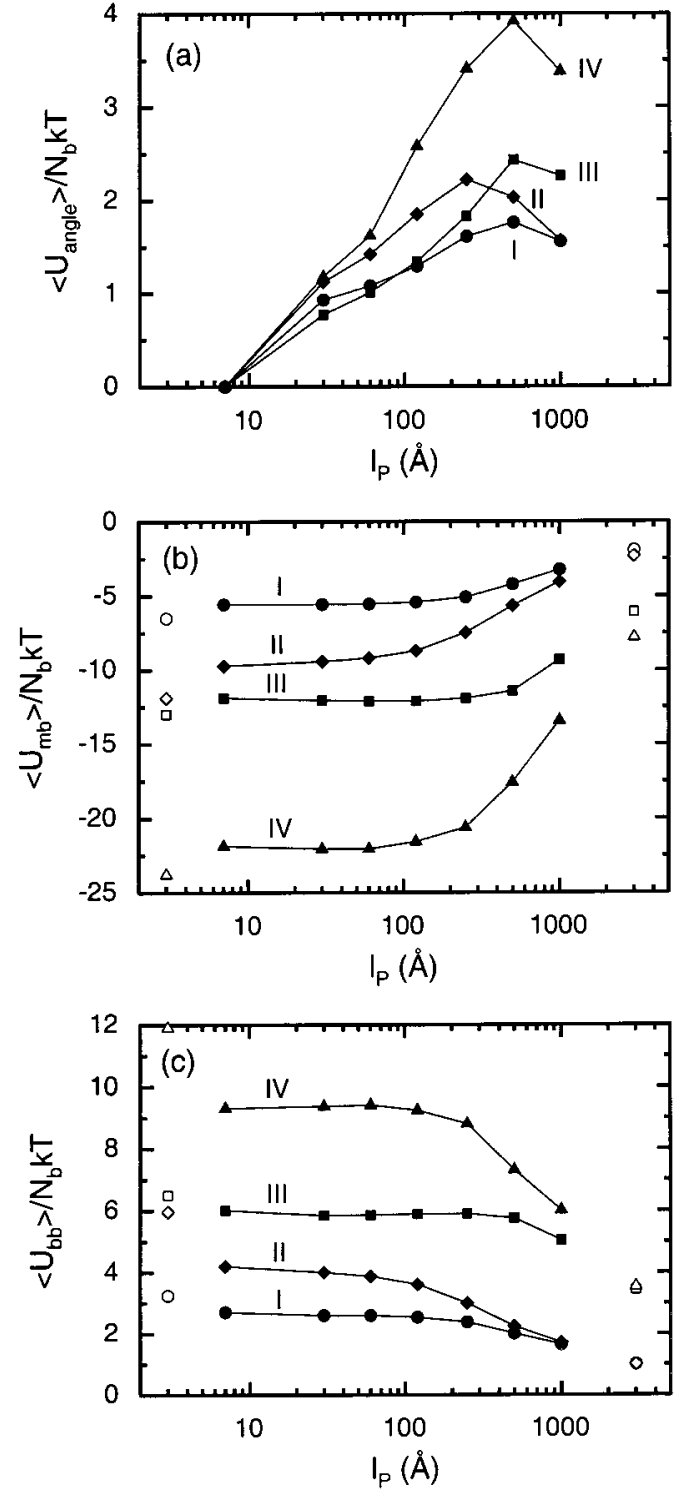

Figure 6. Average and reduced (a) angular $\left(\left\langle\mathrm{U}_{\text {angle }} / \mathrm{N}_{\mathrm{b}} \mathrm{kT}\right\rangle\right)$, (b) electrostatic macroion-bead $\left(\left\langle\mathrm{U}_{\mathrm{mb}} / \mathrm{N}_{\mathrm{b}} \mathrm{kT}\right\rangle\right)$, and (c) el ectrostatic bead-bead interactions $\left(\left\langle\mathrm{U}_{\mathrm{bb}} / \mathrm{N}_{\mathrm{b}} \mathrm{kT}\right\rangle\right)$ as a function of the bare persistence length $\left(I_{p}\right)$ for indicated systems (filled symbols). The lines are only guide for the eye. In (b) and (c), the limiting interaction energies of a fully collapsed chain at the macroion surface (open symbols, left) and of a rigid rod touching the macroion (open symbols, right) are also given (see text for further details). Estimated errors are smaller than the size of the symbols.

smeared out at $r=R_{m}+R_{b}$. The second model involves a rigid rod touching the macroion at the center of the rod, and $\mathrm{U}_{\mathrm{mb}} / \mathrm{N}_{\mathrm{b}} \mathrm{kT}$ and $\mathrm{U}_{\mathrm{bb}} / \mathrm{N}_{\mathrm{b}} \mathrm{kT}$ were calculated numerically by direct summation using rms bead-bead separations from the simulations with the largest bare persistence length (see Table 2).

Figure 6a shows that the average angular energy initially increases as the bare persistence length is increased for all systems. We recall that the variation of the bare persistence length was achieved by altering the force constant of the angular potential energy term given by eq 5 . However, at some (critical) stiffness the angular potential energy starts to decay, and the location of the maxima appear at $600,330,700$, and 620 $\AA$ (from harmonic fits) for systems I-IV, respectively. The angular energy at the critical stiffness is smallest $\left(\approx 1.8 \mathrm{kT} / \mathrm{N}_{\mathrm{b}}\right)$ for system I appearing when the polyion form a single, extended loop and largest $\left(\approx 4 k T / N_{b}\right)$ for system IV occurring when the polyion form a short solenoid (see Figure 1). We notice that the electrostatic macroion-polyion attraction at contact is smallest for system I and largest for system IV.

Figure $6 \mathrm{~b}$ shows that the average electrostatic macroion-bead interaction varies between ca. - 3kT and $-22 \mathrm{kT}$. Bel ow $\mathrm{I}_{\mathrm{P}} \approx 100 \AA,\left\langle\mathrm{U}_{\mathrm{mb}}\right\rangle$ is nearly constant, but at larger $I_{p}$ it starts to increase. A comparison between $\left\langle U_{\mathrm{mb}}\right\rangle$ obtained from the flexible chain and the simplified collapsed model shows that the former attains ca. 80$90 \%$ of the value of the latter. The smallest differences appear for systems I and III with the larger macroion. Similarly, we found that $\left\langle U_{\mathrm{mb}}\right\rangle$ approaches its rod-limit as $I_{p}$ is increased.

The average electrostatic bead-bead repulsion is given in Figure $6 \mathrm{c}$. Also, $\left\langle\mathrm{U}_{\mathrm{bb}}\right\rangle$ remains fairly constant up to $I_{p} \approx 100 \AA$, although $\left\langle U_{b b}\right\rangle$ displays a gradual decrease already at small $I_{p}$ in systems I and II. The comparison of $\left\langle\mathrm{U}_{\mathrm{bb}}\right\rangle$ from the flexible chain and the collapsed model displays here a close agreement for systems I and III and a considerable relative deviation $(>25 \%)$ for systems II and IV. As IP increases, $\left\langle U_{b b}\right\rangle$ approaches its rod limit.

Thus, energetically, the polyion sacrifices angular energy to maintain the favorable electrostatic energy as the angular force constant is increased. However, at some critical point, the bending energy becomes too large, and at this point the detachment of train beads accelerates. The bending energy at the critical point increases as the el ectrostatic macroion-bead attraction is increased.

\section{Discussion}

Our main findings will here be discussed across the different systems and related to recent theoretical developments and MC simulations.

4.1. Flexible Polyion. System I with the lower linear charged and longer polyion and the larger macroi on $\left(Z_{b}\right.$ $=0.5, N_{b}=80$, and $R_{m}=20 \AA$ ) possesses the weakest electrostatic polyion-macroi on interaction $\left(\Gamma_{\mathrm{mb}}=6.50\right)$. The polyion length is ca. 3 times the circumference of the macroion, and the bead coverage of the macroion surface is 0.2 (see Table 2 for definitions). With the fully flexible chain, the polyion forms a complex where the chain is locally folded, with short loops directed away from the surface, making the charge ratio $\left|Z_{p}^{c} / Z_{m}\right|$ drop to ca. 0.7 . The low bead coverage makes the coverage of the macroion surface heterogeneous.

The specific parameters of system II are $\mathrm{Z}_{\mathrm{b}}=0.5, \mathrm{~N}_{\mathrm{b}}$ $=80$, and $R_{m}=10 \AA$. As compared to system I, the macroion size is reduced by a factor of 2 , giving an increased electrostatic interaction $\left(\Gamma_{\mathrm{mb}}=11.9\right)$, an increased polyion length to circumference length ratio to 6 , and a bead coverage of 0.8 . The bond coverage, which also is an appropriate measure of the surface coverage for chains with fixed or nearly fixed bond separation, exceeds unity (Table 2). Figure 1 (row 1, column 2) clearly illustrates full surface coverage and the inability for all beads to be in direct contact with the macroion surface. A large amount of the beads form loops due to lack of space at the surface, and conse quently the charge ratio is substantially reduced $\left(\left|Z_{\mathrm{p}}^{\mathrm{c}} / Z_{\mathrm{m}}\right|=0.78\right)$. The energy analysis also showed that system II is the one deviating most from having a fully collapsed bead layer. 
System III is characterized by $\mathrm{Z}_{\mathrm{b}}=1, \mathrm{~N}_{\mathrm{b}}=40$, and $R_{m}=20 \AA$. The increase of the linear charge density and reduction of the chain length with a factor of 2 as compared to system I leads also to a stronger el ectrostatic polyion-macroion interaction $\left(\Gamma_{\mathrm{mb}}=13.0\right)$. In this system, the flexible chain is again folded, but as compared to system I, the stronger electrostatic interaction with the macroion makes the bead layer thinner, and the shorter chain is only able to make one turn around the macroion. As in system I, large areas of the macroion are uncovered, and the charge ratio $\left|Z_{\mathrm{p}}^{\mathrm{c}} / \mathrm{Z}_{\mathrm{m}}\right|$ increases to 0.93 .

System IV possesses the strongest el ectrostatic interaction $\left(\Gamma_{\mathrm{mb}}=23.8\right)$ achieved by combing the high linear charge density and the small macroion $\left(Z_{b}=1\right.$, $\mathrm{N}_{\mathrm{b}}=40$, and $\mathrm{R}_{\mathrm{m}}=10 \AA$ ). The polyion length is again ca. 3 times the circumference of the macroion and the bead and bond coverages amount to 0.4 and 0.7 , respectively. Although possessing a high surface coverage, the strong macroion-bead interaction is able to bring all beads near the surface and form a monolayer of beads without any protruding loops. The different turns around the macroions are spatially correlated, but the chain is still locally folded. The charge ratio $\left|Z_{p}^{c} / Z_{m}\right|$ is nearly 1.0 .

4.2. Nearly F lexible Chains. As expected, structural rearrangements appear as the chain stiffness is increased. Already a moderate increase of the chain stiffness has consequences on the complex. The suppression of the local folding by a weak angular potential increasing the bare persistence length from 7 to 30-60 $\AA$ enables the beads to approach closer to the macroion as deduced form, e.g., the reduced $r c n$ and the charged ratio $\left|Z_{p}^{C} / Z_{m}\right|$. Obviously, for a fully flexible chain, the electrostatically driven collapse is partly counteracted by chain configuration entropy. System II constitutes however an exception, since the macroion area is not sufficiently large to house all beads. In system III, the chain attains an incomplete "tennis ball seam" conformation (the chain is too short to make a full seam), whereas in system IV with the smaller macroion a three-turn solenoid is formed. In systems III and IV, essentially no tails appear yet.

4.3. Stiffer Chains. When the stiffness of the chains is increased further, additional structural rearrangements appear. The difference in the macroion size becomes less important as compared to the difference in chain linear charge density (and length). As the bare persistence length exceeds the macroi on curvature, the angular potential starts to markedly affect the ability of the chain to be collapsed at the macroion.

In systems I and II, more extended loops are formed, and in particular in system II with the smaller macroion and stronger bead-macroion interaction "rosette"-like structures are formed. At even larger bare persistence lengths, the angular energy exceeds the gain in electrostatic energy of forming many chain-macroion contacts. At this stage the chain releases bending tension by reducing the number of contact points and becomes straighter. Finally, with an even larger bare persistence length (or weaker electrostatic interaction) than considered here, the polyions would straighten out even further and only a single polyion-macroion contact region would appear as the equilibrium configuration.

The structural developments in systems III and IV are however different. In system III, the chain is straightened further, albeit still being collapsed at the macroion surface by forming a one and half turn around the macroion. First with $I_{p}=500 \AA$, substantial tails are formed and with the stiffest chain considered, a U-turn involving still half of the chain is formed. In system IV, the ends of the three-turn solenoid start to detach as the stiffness is increased. Between $I_{p}=500$ and $1000 \AA$ there is a transition to a singleloop conformation. At this stagethe bending tension becomes too large to keep the chain fol ded around the macroion. With an even larger stiffness, also here a single polyion-macroion contact region would appear.

4.4. Relation to Theories and Previous Model Simulations. Despite the large number of theories developed to describe the polyion-macroion complexation, yet not a single theory covers the rich complex behavior explored here by MC simulations.

The collapsed state of flexible polyions has been examined by a number of theories, ${ }^{20-22,26,33}$ and in particular the aspect of overcharging has been considered. However, bead-bead excluded-volume effects are generally ignored, and the present study involves a system with a high surface coverage (system II), where such an effect plays a significant role. We have also demonstrated the effect of the chain configurational entropy and showed that the strongest complexation is obtained with a semiflexible chain. This is not too surprising, since the adsorption of polyions to an oppositely charged plane improves with increasing chain stiffness.

The spatial arrangements of semiflexible chains interacting with a small spherical center through a short-range attractive force have theoretically been treated by Schiessel et al. ${ }^{25}$ They employed a wormlike chain model, which describes the chain as a semiflexible tube characterized by a bending and a torsional stiffness. The theoretically treatment required them to consider a ring polymer, but the results are not claimed to depend critically on an opening of the ring. ${ }^{25} \mathrm{At}$ low bending stiffness and at not too attractive chain-sphere interaction, their theory predicts a "rosette"-like structure (see Figure 1 of ref 25) with up to five loops and with the number of loops reducing as the bending stiffness was increased. Both the appearance of loops and the reduction of the number of them are in qualitati vely agreement with our predictions for system II. Moreover, the theory predicts a first-order transition to a compact wrapping state (see Figure 2 of ref 25) as the attraction between the tube and the center is increased for a given bending stiffness. Similar transition is found here as the linear charge density of the polyion is increased (see Figure 1, row 3 , column $2 \rightarrow$ column 4), but the location of this transition has not here been investigated in further detail.

Recently, Netz and J oanny modeled the polyionmacroion complexation using a continuous polymer model containing bending energy, repulsive el ectrostatic intrachain interaction, and attractive electrostatic interaction between the polymer and the macroion. ${ }^{23}$ The ground state approximation was invoked making the theory only applicable for bare persistence lengths larger than the size of the macroion. Moreover, the polymer was restricted to a common plane with the macroion center, and the electrostatic interaction was treated on the Debye-Hückel level. Of interest here, they derived scaling relations for the "touching" and "wrapping" transitions. The former transition was defined as the point where the chain locally attain the 
curvature of the macroion and the latter where the length of the wrapping polyion segment approaches the radius of the macroion. Owing to (i) different polymer models and (ii) screened vs no screened electrostatic interaction detailed comparison is impeded. Nevertheless, for a constant screening and flexibility Netz and J oanny predicted transitions from point contact, loosely wrapped, and to tightly wrapped complexes as the macroion charge is increased (F igure 5 of ref 23), similar to our observations and conclusions.

Finally, Chodanowski and Stoll have performed extensive MC simulation of the complexation between one polyion and an oppositely charged macroion interacting via the screened Debye-Hückel potential using a freely jointed hard-sphere chain. ${ }^{28,29}$ Their studies involved simulation of one chain with varying chain lengths (25-200 beads with fixed linear charged density $-1 \mathrm{e} / 7.14 \AA)$ and one macroi on $\left(R_{m}=17.85 \AA\right.$ and $Z_{m}=$ $50)^{28}$ and one chain (100 beads) and one macroion with variable size and charge at fixed surface charge density, ${ }^{29}$ both cases at different screening lengths (0-1 M 1:1 salt) and were focused on the adsorption/desorption transition and the structure of the complexes. The macroion size and linear charge density used 28 are embraced by the values used here, and at those few conditions where our model parameters are similar (their $N_{b} \approx 50$ ), the obtained wrapping structure is similar. From their simulations with variable screening length, they concluded that the adsorption/desorption transition appears at a macroion-bead interaction of $\approx 1 \mathrm{kT}$, supporting the present observation of strong complexation.

\section{Conclusion}

On the basis of Monte Carlo simulations, an examination of the complexation between a single polyion and a single, oppositely charged, and spherical macroion has been performed. Relevant physical parameters have been discussed, and focus has been made on how the bare persistence length affects the structure and energetics. This was made for some combinations of polyion linear charge densities and macroion sizes at constant and equal absolute polyion and macroion charge without salt applied to aqueous solution at ambient temperature. Despite the selected strong polyion-macroion interaction $(Q(10 \mathrm{kT} / \mathrm{bead}))$ and the concomitant large energy barriers between different states, equilibrium results were obtained by combining different types of trial moves and in some cases considering different initial configurations.

The flexible chains displayed a collapsed state with the chains wrapped around the macroion but still locally folded. The maximal collapse appeared for a semiflexible chain. The fully flexible chains displayed a locally more folded configuration opposing an optimal collapse. A large range of structures was encountered as the chain stiffness gradually was increased. The collapsed state appearing with a flexible chain was successively transformed into "tennis ball seam", solenoid, multiloop ("rosette"-like), single-loop, and U-turn structures, however not all of them present in the same system. The number of beads in a single loop and tail was increased as the chain stiffness was increased, whereas the number of beads in a single train displayed a maximum at intermediate stiffness. In the present systems with equal absolute charge of the macromolecules the macroion prefer to complex to the central part rather than to one end of the polyion (symmetric complexation).

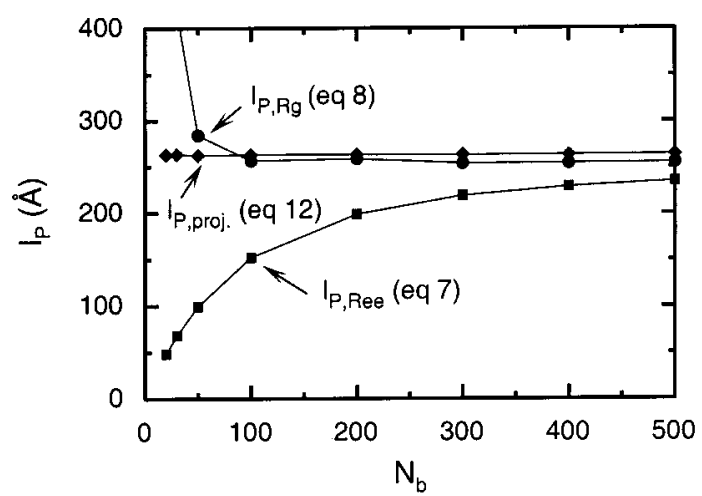

Figure 7. Bare persistence length $\left(I_{P}\right)$ as a function of the number of beads $\left(\mathrm{N}_{\mathrm{b}}\right)$ of an uncharged chain of $\mathrm{N}_{\mathrm{b}}$ beads joined by a harmonic bond potential given by eq 4 with $\mathrm{k}_{\text {bond }}=0.4$ $\mathrm{N} / \mathrm{m}$ and $\mathrm{r}_{0}=5.0 \AA$ and by a harmonic angle potential given by eq 5 with $k_{\text {angle }}=35 \mathrm{~J} /(\mathrm{mol} \mathrm{deg})$ and $\alpha_{0}=180^{\circ}$. The bare persistence length was evaluated using indicated equations.

The structure of the present system is primarily controlled by (i) the attractive polyion-macroion interaction favoring the complexation and (ii) the repulsive bead-bead interaction and the bending interaction opposing the complexation. The present study gives additional insight into the magnitudes of these interaction terms as well as the competition among them.

Although quantitative comparison with recent theoretical developments is no yet trivial due to different model systems and features studied, important structures previously predicted have here been confirmed.

Acknowledgment. A.A. thanks Andrei Broukhno and Magnus Ullner for helpful discussions. Financial support from the Swedish National Research Council (NFR) is gratefully acknowledged.

\section{Appendix}

A short account of different approaches to evaluate the persistence length of a chain composed of a sequence of beads and a motivation of our choice are given here.

The persistence length $I_{p}$ is used as a measure of the chain stiffness, and it has been defined and evaluated in several different ways. ${ }^{39,40} \mathrm{U}$ sing global chain properties, the persistence length can be defined by

$$
I_{P, R_{e e}}=\frac{R_{e e}^{2}}{2\left(N_{b}-1\right) b}+\frac{b}{2}
$$

where $\mathrm{R}_{\mathrm{ee}}$ is the end-to-end distance, $\mathrm{N}_{\mathrm{b}}$ the number of beads in the chain, and $b$ the bond length. In another gl obal approach based on the wormlike chain model, IP is given by

$$
R_{G}^{2}=\frac{L I_{P, R_{G}}}{3}-I_{P, R_{G}}^{2}+2 \frac{I_{P, R_{G}}^{3}}{L}-2 \frac{I_{P, R_{G}}^{4}}{L^{2}}\left(1-e^{\left.-L / I_{P, R_{G}}\right)}\right.
$$

with the limiting value

$$
\mathrm{I}_{\mathrm{P}, \mathrm{R}_{\mathrm{G}}} \rightarrow 3 \mathrm{R}_{\mathrm{G}}^{2} / \mathrm{L}
$$

as $L / /_{P, R_{G}} \rightarrow \infty$, where in eqs 8 and $9 R_{G}$ denotes the radius of gyration and $L=\left(N_{b}-1\right) b$ the polymer contour length. Since $L / /_{p, R_{G}}$ is typically large in experimental systems and $R_{G}$ is readily available from e.g. scattering experiments, eq 9 is often used when report- 
ing flexibility of experimental polymer systems. Equations 7 and 9 are equival ent for a Gaussian chain where $\left\langle\mathrm{R}_{\mathrm{G}}^{2}\right\rangle=\left\langle\mathrm{R}_{\mathrm{ee}}^{2} / 6\right.$.

In a third approach, the persistence length is based on the projection of angles between bonds vectors according to

$$
\mathrm{I}_{\mathrm{P}, \mathrm{proj}}=\mathrm{b} \sum_{\mathrm{k}}\left\langle\cos \theta_{\mathrm{k}}\right\rangle
$$

where $b$ is again the bond length and $\theta_{\mathrm{k}}$ is the angle between two bond vectors separated by $\mathrm{k}$ bonds. $\mathrm{F}$ or a freely rotating chain with equal and fixed angles, the average of directional cosines becomes $\left\langle\cos \theta_{\mathrm{k}}\right\rangle=$ $\cos ^{k} \theta_{1}$, where $\theta_{1}$ is the first directional angle. The sum in eq 10 becomes a geometric series, and for an infinite chain we obtain

$$
\mathrm{I}_{\mathrm{P}, \mathrm{proj}}=\frac{\mathrm{b}}{1-\cos \theta_{1}}
$$

In the case of fluctuating bond length and bond angle, we substitute $\mathrm{b}$ by $\left\langle\mathrm{R}_{\mathrm{bb}}^{2}\right\rangle^{1 / 2}$ and $\cos \theta_{1}$ by $\left\langle\cos \theta_{1}\right\rangle$. Moreover, the substitution $\theta_{1}=\pi-\alpha$ leads to the following expression

$$
I_{P, \text { proj }}=\frac{\left\langle R_{b b}^{2}\right\rangle^{1 / 2}}{1+\langle\cos \alpha\rangle}
$$

Figure 7 shows the persistence length of an uncharged chain of beads connected by harmonic bonds as obtained using eqs 7, 8, and 12 as a function of the number of beads using $\mathrm{k}_{\text {angle }}=35 \mathrm{~J} /\left(\mathrm{mol} \mathrm{deg}{ }^{2}\right)$. The persistence length of all three approaches appears to converge to $I_{P}$ $\approx 260 \AA$ as $N_{b}$ is increased; however, the rate of convergence differs. The route using the end-to-end separation has the slowest convergence (still not converged at $\mathrm{N}_{\mathrm{b}}=500$ or $\mathrm{L} \approx 10 \mathrm{l}_{\mathrm{p}}$ ) and approaches its limit from bel ow. The use of the radius of gyration converges much faster. Finally, the local approach is, as expected, least sensitive on the chain length. Since, in this study very rigid chains with $I_{p} \gg L$ are considered, the local approach of calculating the persistence length has been adopted. Here, we do not concern ourselves about the interpretations of the different methods of evaluating persistence lengths. ${ }^{39,40}$

\section{References and Notes}

(1) Goddard, E. D. Colloids Surf. 1986, 19, 255.

(2) Polymer-Surfactant Systems; Kwak, J. C. T., Ed.; Marcel Dekker: New York, 1998; Vol. 77
(3) Lindman, B.; Thalberg, K. Polymer-surfactant interactionsrecent developments. In Interactions of Surfactants with Polymers and Proteins; Goddard, E. D., Ananthapadmanabhan, K. P., Eds.; CRC Press: Boca Raton, FL, 1993; p 203.

(4) Hansson, P.; Lindman, B. Curr. Opin. Colloid Interface Sci. 1996, 1, 604.

(5) Doublier, J. L.; Garnier, C.; Renard, D.; Sanchez, C. Curr. Opin. Colloid Interface Sci. 2000, 5, 202.

(6) Shubin, V.; Samoshina, Y.; Menshikova, A.; Evseeva, T. Colloid Polym. Sci. 1997, 275, 655

(7) Shubin, V. J . Colloid Interface Sci. 1997, 191, 372.

(8) Marques, E. F.; Regev, O.; Khan, A.; Miguel, M. D.; Lindman, B. Macromolecules 1999, 32, 6626.

(9) Mashl, R. J .; Gronbech-J ensen, N.; Fitzsimmons, M. R.; Lutt, M.; Li, D. Q. J . Chem. Phys. 1999, 110, 2219.

(10) Wang, Y. L.; Kimura, K.; Dubin, P. L.; J aeger, W. Macromolecules 2000, 33, 3324.

(11) Wang, Y. L.; Dubin, P. L.; Zhang, H. W. Langmuir 2001, 17 1670.

(12) Zhang, H. W.; Ohbu, K.; Dubin, P. L. Langmuir 2000, 16, 9082.

(13) Fuchs, A.; Killmann, E. Colloid Polym. Sci. 2001, 279, 53.

(14) von Goeler, F.; Muthukumar, M. J . Chem. Phys. 1994, 100, 7796.

(15) Wallin, T.; Linse, P. Langmuir 1996, 12, 305.

(16) Wallin, T.; Linse, P. J . Phys. Chem. 1996, 100, 17873.

(17) Wallin, T.; Linse, P.J. Phys. Chem. B 1997, 101, 5506.

(18) Wallin, T.; Linse, P. J . Chem. Phys. 1998, 109, 5089.

(19) Kong, C. Y.; Muthukumar, M. J. Chem. Phys. 1998, 109, 1522.

(20) Gurovitch, E.; Sens, P. Phys. Rev. Lett. 1999, 82, 339.

(21) Park, S. Y.; Bruinsma, R. F.; Gelbart, W. M. Europhys. Lett. 1999, 46, 454 .

(22) Mateescu, E. M.; J eppesen, C.; Pincus, P. Europhys. Lett. 1999, 46, 493

(23) Netz, R. R.; J oanny, J . F. Macromolecules 1999, 32, 9026.

(24) Kunze, K. K.; Netz, R. R. Phys. Rev. Lett. 2000, 85, 4389.

(25) Schiessel, H.; Rudnick, J .; Bruinsma, R. F.; Gelbart, W. M. Europhys. Lett. 2000, 51, 237.

(26) Nguyen, T. T.; Shklovskii, B. I. Physica A 2001, 293, 324

(27) N guyen, T. T.; Shklovskii, B. I. J . Chem. Phys. 2001, 114 5905.

(28) Chodanowski, P.; Stoll, S. Macromolecules 2001, 34, 2320.

(29) Chodanowski, P.; Stoll, S. J. Chem. Phys. 2001, 115, 4951.

(30) Carlsson, F.; Linse, P.; Malmsten, M. J . Phys. Chem. B 2001 105,9040

(31) J onsson, M.: Linse, P. J . Chem. Phys. 2001, 115, 3406.

(32) J onsson, M.; Linse, P. J . Chem. Phys. 2001, 115, 10957

(33) Schiessel, H.; Bruinsma, R. F.; Gelbart, W. M.J . Chem. Phys. 2001, 115, 7245 .

(34) J onsson, M.; Linse, P. Phys. Rev. E, submitted for publication

(35) Linse, P.; Piculell, L.; Hansson, P. Models of polymersurfactant complexation. In Polymer-Surfactant Systems; Kwak, J. C. T., Ed.; Marcel Dekker: New York, 1998; Vol. 77.

(36) J eppesen, C.; Mateescu, E. M.; Pincus, P. Private communication

(37) Computer Simulation of Liquids; Allen, M. P., Tildesley, D. J., Eds.; Oxford University Press: New York, 1987.

(38) MOLSI M, Ver. 3.0, Linse, P., Lund University, Sweden, 2000

(39) Ullner, M.; J onsson, B.; Peterson, C.; Sommelius, O.; Söderberg, B. J . Chem. Phys. 1997, 107, 1279.

(40) Ullner, M.; Woodward, C. E. Macromol ecules 2002, 35, 1437. 